\title{
Whole and Part in Mathematics ${ }^{1}$
}

\author{
John L. Bell \\ University of Western Ontario
}

\begin{abstract}
The centrality of the whole/part relation in mathematics is demonstrated through the presentation and analysis of examples from algebra, geometry, functional analysis, logic, topology and category theory.

Key words. Part, whole, mereosis, Stone duality, Gelfand duality, Grothendieck topology.
\end{abstract}

The relationship between whole and part—what I shall call mereosis-has played a central role in the development of mathematics. Perhaps its first explicit appearance is in the fifth axiom (as opposed to postulate) laid down by Euclid in his immortal "Elements", to wit, the whole is greater than the part. Cantor's repudiation of this thesis more than 2000 years later was a pivotal element in his transfinite set theory.

A classic example of mereosis is to be found in analytic geometry. Here arbitrary points or vectors in $n$-dimensional Euclidean space $\mathbb{R}^{n}$ are represented as linear combinations of $n$ basis vectors $\left\{\mathbf{e}_{1}, \ldots, \mathbf{e}_{n}\right\}$. Here the "whole" $\mathbb{R}^{n}$ is completely determined ("coordinatized") by its (very small) part $\left\{\mathbf{e}_{1}, \ldots, \mathbf{e}_{n}\right\}$. This example has, of course, been extended to the concept of a basis for a vector space, and to the general idea of a mathematical structure being generated by a part, in much the same way as a written language is generated by an alphabet.

A related example arises with the observation that any point in a convex polygon or polyhedron $P$ is the centre of mass or barycentre of a unique

\footnotetext{
${ }^{1}$ Revised and expanded version of a paper delivered at the conference "Wholes and their Parts" held in Bolzano, Italy, in June 1998. I am most grateful to Alberto Peruzzi and Roberto Poli for organizing a truly memorable meeting.
} 
distribution of masses placed at the vertices of $P$. This fact is generalized to Choquet's theorem, an important result of functional analysis (see, e.g. Edwards 1965). This theorem asserts that any point of a compact convex subset $C$ in a normed space is the barycentre (in a suitably extended sense) of a measure living on the set $E$ of extreme points of $C$. (Here an extreme point of $C$ is any point not contained in the interior of a line segment in C.) An important consequence of this is the Krein-Milman theorem: $C$ is the least closed convex set containing $E$. Both of these theorems show that the "whole" $C$ is completely determined in a suitable sense by its small part $E$.

A "part" of a mathematical whole may be obtained by ignoring some aspect of its structure. In this sense, for example, the additive group of integers is part of the ring of integers. Sometimes a part taken in this sense may determine a whole. For instance, the purely algebraic part of the ordered field of real numbers uniquely determines its ordering ( $r \leq s$ iff $s-r$ is a square) and thereby the whole structure. This obvious fact has a nontrivial generalization (a portion of the Artin-Schreier theorem): any real-closed field $F$ (i.e. such that -1 is the sum of squares in any proper algebraic extension of $F$, but not in $F$ itself) has a unique total ordering compatible with its algebraic structure.

A mathematical whole may be determined by a part in the sense that each member of the whole is "approximable" in a suitable (i.e., topological) sense by members of the part. The classic example of this is the fact that each real number is the limit of a sequence of rational numbers. This fact of course has deep roots in mathematical history. The Pythagoreans around 550 B.C. apparently believed that the whole corpus of "ratios" of similar geometric magnitudes was identical with the part consisted of "ratios" of whole numbers (so that measurement is always reducible to counting). Their discovery of the incommensurablity of the side and diagonal of a square (i.e. the irrationality of $\sqrt{ } 2)$ - the first "mathematical crisis" - forced them and their mathematical successors to recognize that whole number ratios constitute only a proper part of that whole, and so to formulate a workable description of that whole: this is 
Eudoxus's theory of proportions.

Other important examples of this sort of mereosis are provided by the Weierstrass approximation theorems, namely, any continuous real or complex continuous function on a closed real interval is the uniform limit of a sequence of polynomials; and any $2 \pi$-periodic complex function on the real line is the uniform limit of a sequence of trigonometric polynomials. In both of these cases the respective wholes (arbitrary continuous or periodic functions) are determined through uniform approximation or "variation" along a specified part (polynomials or trigonometric polynomials). These $19^{\text {th }}$ century theorems were generalized in the $20^{\text {th }}$ century to the Stone-Weierstrass theorem (see, e.g., Edwards 1965) which gives a criterion for a part $A$ of an algebra of continuous real- or complex-valued functions on a compact Hausdorff space $X$ to be identical with the whole algebra of such functions: to wit, $A$ is a closed selfconjugate subalgebra containing the unit function and separating the points of $X$.

Galois theory (see, e.g, Bourbaki 1979) provides a far-reaching instance of mereosis in mathe-matics. In its classical setting, we are given a polynomial equation $f(x)=0$ over a field $F$ (typically, the rational numbers), and we seek a solution to this equation by "radicals", that is, the extraction of $n^{\text {th }}$ roots for arbitrary $n$. The pertinent "whole" in this case is the so-called splitting field $K$ of $f$ over $F$, that is, the least field containing $F$ in which $f$ can be completely factorized into linear factors. The condition for solubility by radicals of $f$ can be formulated as a condition on the way in which the whole field $K$ (to be strictly precise, some well-determined extension of $K$ ) can be built up from suitable "parts"-in this case, intermediate subfields $L$ of $K$ for which $F \subseteq L \subseteq K$. Associated with $K$ is a group $G$-its Galois group - which is isomorphic to a subgroup of the group of permutations of $n$ objects, where $n$ is the degree of $f$. The group G constitutes a new "whole"; its pertinent "parts" are its subgroups. The fundamental theorem of Galois theory asserts that the assemblages of parts of the two wholes $K$ and $G$ are, in a suitable sense, isomorphic. (The 
correspondence establishing the isomorphism is an instance of a very general sort of correspondence now called a Galois connection or adjunction.) This isomorphism has the effect of transforming the complex property of the assemblage of parts of the whole $K$ expressing the solubility by radicals of $f$ into a comparatively simple property - solvability —of the parts of $G$ (and hence of $G$ itself). Here we have an instance of the mereotic pattern

\section{WHOLE $\rightleftarrows$ PARTS}

There are some important examples of mereosis in mathematical logic. To begin with, we have the evident, but often useful fact that the consistency of a first order theory is reducible to the consistency of its finite parts. Then there is the indispensable compactness theorem (see e.g. Bell and Slomson 1969) which asserts that a first-order theory $\Sigma$ has a model precisely when each finite part of $\Sigma$ does. Here the model of $\Sigma$ can be fashioned directly (as an "ultraproduct") from the models of the finite parts of $\Sigma$. This is an instance of "synthesis" of a mathematical structure from parts-a procedure we shall return to.

The celebrated Löwenheim-Skolem theorem asserts that the definable properties of a given (infinite) structure are identical with those of some of its (comparatively small) parts and with those of other, larger, structures, of which the original constitutes a (small) part. A related result, the reflection principle of set theory (see, e.g., Bell and Machover 1977) asserts that the definable properties of the universe of sets is "reflected" in those of a small part-to wit, a set.

Conservation results in mathematical logic are also a source of examples of merosis. Here we are given theories $\Sigma_{1} \subseteq \Sigma_{2}$ formulated in languages $\mathscr{L}_{1} \subseteq \mathscr{L}_{2}$ respectively. $\Sigma_{2}$ is a conservative extension of $\Sigma_{1}$ if the part of $\Sigma_{2}$ consisting of $\mathscr{L}_{1}$ sentences is identical with $\Sigma_{1}$. The basic instance of this arises when $\Sigma_{1}$ is any theory in a first-order language $\mathscr{L}_{1}$ and $\Sigma_{2}$ is the set of logical consequences of 
$\Sigma_{1}$ in a first-order language $\mathscr{L}_{2} \supseteq \mathscr{L}_{1}$. Abraham Robinson's Nonstandard Analysis (Robinson 1996) provides another example: here $\Sigma_{1}$ is classical analysis formulated in the usual set-theoretic language $\mathscr{L}_{1}$ and $\Sigma_{2}$ is nonstandard analysis formulated in the appropriately extended language $\mathscr{L}_{2}$. A further example was provided by Gödel: here one takes $\mathscr{L}_{1}$ to be the language of (settheoretic) arithmetic, $\Sigma_{1}$ to be the set of statements of $\mathscr{L}_{1}$ provable in ZermeloFraenkel set theory $\mathbf{Z F}, \mathscr{R}_{2}$ the language of set theory and $\Sigma_{2}$ the theory $\mathbf{Z F}+$ the axiom of choice + the continuum hypothesis. As a failed example we may consider Hilbert's program in its original form. Here $\mathscr{L}_{1}$ is the language of "concrete" mathematics, $\Sigma_{1}$ the "concretely" provable statements of $\mathscr{L}_{1}, \mathscr{L}_{2}$ the language of "ideal" mathematics, and $\Sigma_{2}$ the "ideally" provable statements of $\mathscr{L}_{2}$. Hilbert hoped to prove $\Sigma_{2}$ a conservative extension of $\Sigma_{1}$ but, as is well-known, Gödel showed, through his famous incompleteness theorems, that this could not be the case.

Our first two examples of mereosis showed how a mathematical structure can be determined by a single specially chosen part. We now consider situations in which such structures can be reconstituted from a suitably chosen plurality of parts.

A simple instance of this is the fact that any object $A$ in a category of algebras can be recaptured from its finitely generated subalgebras: $A$ is the colimit of the diagram whose vertices are the finitely generated subalgebras of $A$ and whose arrows are inclusion maps on these vertices.

More interestingly, mathematical structures can often be reconstituted from parts which arise as "level sets" of "quantities" varying over them in a continuous manner (just as a surface can be mapped by level curves). The archetypal example here is that of a compact Hausdorff space $X$. In this case $X$ can be recaptured from its continuous zero-sets $Z(f)$ : here $Z(f)$ is the part of $X$ on which the continuous real-valued function $f$ vanishes (Gillamn and Jerison 1960). Each $Z(f)$ may be thought of as a piece of information about $X$ identifying 
the part of $X$ over which the continuously varying quantity $f$ assumes a particular constant value (in this case, zero). In this sense zero-sets constitute informative parts of $X$. (If $X$ is a surface in 3-space then the relevant continuous zero-sets-the level curves - are those arising as intersections of the surface with planes parallel to one of the coordinate planes.) We find that maximal nondisjoint sets of informative parts of $X$ correspond to points of $X$, and that $X$ can then be recaptured by imposing a natural topology on the collection of such sets of informative parts. In this case then, "if you know the informative parts, you know the whole."

The technique of reconstituting a space from its informative parts extends to algebraic structures such as (commutative) rings which arise by abstracting the idea of (continuously) varying quantity. Here the idea is to assemble informative parts of a given ring $R$ into a topological space $X$, and to identify a (topological) ring $R^{*}$ resembling $R$ in certain key respects, in such a way that $R$ may be identified as the ring of continuous $R^{*}$-valued maps on $X$.

There are two classical examples of this.

First, Stone duality (see Johnstone 1982). In this case $R$ is a Boolean ring $B$-i.e., one in which the identity $x^{2}=x$ holds. (The concept of Boolean ring was abstracted, essentially by Boole himself, from the idea of propositional or truth function, viz., a varying "quantity" assuming just the two "truth values" true (1) and false (0).) Here the informative parts of $B$ are its maximal ideals-the parts of $B$ on which a homomorphism to the discrete topological ring $\mathbf{2}$ of integers modulo 2 takes value 0 . These can be assembled into a topological space $X-$ the Stone space of $B$-and $B$ then identified as the ring of continuous maps from $X$ to $\mathbf{2}$.

Next, Gelfand duality (see, e.g., Loomis 1953). In this case $R$ is a $C^{*_{-}}$ algebra-a certain kind of Archimedean ordered ring. The informative parts of $R$ are again its maximal ideals-here these are the parts on which a homomorphism to a field vanishes. These are assembled into a topological space $X$ and $R$ then identified as the ring of continuous maps from $X$ to the real 
field $\mathbb{R}$.

In both of these cases the given ring $R$ is recaptured as a ring of continuously variable quantities over a topological space $X$ assembled from certain parts of $R$. More can be done by introducing the category (a topos) $\operatorname{Shv}(X)$ of sheaves over $X$, which may be described as the universe of all objects undergoing continuous variation over $X$ (see, e.g., Mac Lane and Moerdijk 1992). It turns out that any Boolean ring may be identified with the ring 2 constructed in $\operatorname{Shv}(X)$, and any $C^{*}$-algebra with the ring $\mathbb{R}$ constructed in $\operatorname{Shv}(X)$. To put it another way, any Boolean ring $B$ (resp., $C^{*}$-algebra $R$ ) can be regarded as the ring 2 (resp., $\mathbb{R}$ ) undergoing continuous variation over the topological space assembled from its informative parts.

To reconstitute an arbitrary (commutative) ring $R$ in a similar way, the relevant informative parts are prime ideals-parts of $R$ on which a homomorphism to an integral domain vanishes, and these are assembled into a space $X$ called the Zariski spectrum of $X . R$ can then be identified with a certain local ring in $\mathbf{S h v}(X)$ : see Mulvey 1979. (A local ring is one which is almost a field in the sense that, for any element $x$, either $x$ or $1-x$ is invertible: both 2 and $\mathbb{R}$ are local rings, as is the ring of germs of continuous real-valued functions at any point of a topological space.) Thus any commutative ring can be identified as a certain "near-field" undergoing continuous variation over the space assembled from its informative parts.

To sum these cases up:

\section{PARTS + VARIATION $\longrightarrow$ WHOLE}

The concept of part is given a very general formulation in category theory (see Lawvere and Schanuel 1997). Given an object $A$ in a category $\mathscr{C}$, a subobject of $A$ is a monic arrow $\alpha: S \rightarrow A$ in $\mathscr{C}$. The subobjects of $A$ constitute the objects of a category $\operatorname{Sub}(A)$, the category of subobjects of $A$, in which an 
arrow from an object $S \stackrel{\alpha}{\longrightarrow} A$ to an object $T \stackrel{\beta}{\longrightarrow} A$ is a (necessarily monic) arrow $S \stackrel{f}{\longrightarrow} T$ in $\mathscr{C}$ for which the diagram

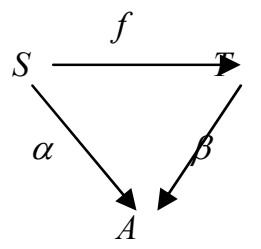

commutes. In $\operatorname{Sub}(A)$ there is at most one arrow between any given pair of objects, so $\operatorname{Sub}(A)$ is a preordered class. A part of $A$ is an equivalence class of subobjects of $A$ under the equivalence relation $\sim$ which identifies two subobjects $S \stackrel{\alpha}{\longrightarrow} A$ and $T \stackrel{\beta}{\longrightarrow} A$ precisely when there is an isomorphism $S \stackrel{i}{\longrightarrow} T$ making the diagram

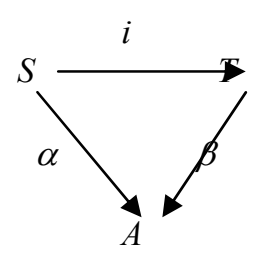

commute. The class $\operatorname{Part}(A)$ of parts of $A$ is then partially ordered by the relation $\subseteq$ of inclusion: writing $\tilde{\alpha}$ for the equivalence class of a subobject $\alpha$, $\tilde{\alpha} \subseteq \tilde{\beta}$ just when there is an arrow $S \stackrel{f}{\longrightarrow} T$ in C making the diagram (*) above commute.

A particularly important kind of mereosis in mathematics is the idea of an object being covered by its parts. In differential geometry, for example, a differentiable manifold is a topological space which is covered in an appropriate way by open parts each of which is homeomorphic to a part of $\mathbb{R}^{n}$. In set theory each set is "covered" by its family of singletons-its one element subsets-in the sense of being the union of that family. In intuitionistic mathematics, where the law of excluded middle is not generally affirmable, individuals are 
"smeared out"-that is, do not necessarily satisfy the condition $a=b$ or $a \neq b$ so that a singleton must be redefined as a set containing at most one element. Objects which unions of singletons in this new sense play the role of "generalized sets".

For sets, the covering relation satisfies the following three characteristic conditions: (i) $\{U\}$ covers $U$, ("each set covers itself"); (ii) if a family $\mathscr{A}$ of sets covers $U$ and $V \subseteq U$, then $\mathscr{A} \mid V=\{A \cap V: A \in\{\}$ covers $V$ ("the restriction of a cover to a part is a cover"); and (iii) if $\mathscr{A}$ covers $U$ and, for each $A \in \mathscr{A}, \mathscr{B}_{A}$ covers $A$, then $\bigcup_{A \in \mathscr{A}} \mathscr{B}_{A}$ covers $U$ ("the union of a covering family of covers is a cover". Grothendieck generalized these conditions to arbitrary categories, defining what came to be known as a Grothendieck topology (see Mac Lane and Moerdijk 1992). This notion assumes a particularly transparent form in the context of partially ordered sets. Thus let $(P, \leq)$ be a fixed but arbitrary partially ordered set: we shall use letters $p, q, r, s, t$ to denote elements of $p$. A subset $S$ of $P$ is said to be a sharpening of, or to sharpen, a subset $T$ of $P$, written $S \prec T$, if $\forall s \in S \exists t \in T(s \leq t)$. A cover scheme on $P$ is a map $\mathbf{C}$ assigning to each $p \in P$ a family $\mathbf{C}(p)$ of subsets of $p \downarrow=\{q: q \leq p\}$, called (C-)covers of $p$, such that, if $q \leq p$, any cover of $p$ can be sharpened to a cover of $q$, i.e.,

$$
S \in \mathbf{C}(p) \& q \leq p \rightarrow \exists T \in \mathbf{C}(q)[\forall t \in T \exists s \in S(t \leq s)] .
$$

This corresponds to condition (ii) above. A Grothendieck topology on $P$ may now be identified as a cover scheme $\mathbf{C}$ on $P$ satisfying counterparts of conditions (i) and (iii) above, namely, $p \downarrow \in \mathbf{C}(p)$ for all $p \in P$ and, if $S \in \mathbf{C}(p)$ and, for each $s \in S, T_{s} \in \mathbf{C}(s)$, then $\bigcup_{s \in S} T_{s} \in \mathbf{C}(p)$.

With each topological space $T$ in the usual sense there is an associated notion of closure: here the closure $\bar{X}$ of a subset $X$ of $T$ is the least closed set containing $X$, i.e. the least set $Y$ containing $X$ with the property that, if every neighbourhood of a point $p$ intersects $Y$, then $p \in Y$. This closure operation satisfies the well-known Kuratowski axioms, namely 


$$
X \subseteq \bar{X}, \quad \overline{\bar{X}}=\bar{X}, \quad \overline{X \cup Y}=\bar{X} \cup \bar{Y} .
$$

Grothendieck topologies also give rise to a kind of closure operation, only on sieves rather than arbitrary subsets: a sieve in a partially ordered set $(P, \leq)$ to be a subset $S$ of $P$ which is $\geq$-stable, that is, satisfies $p \in S \& p \geq q \rightarrow q \in S$. Accordingly suppose given a Grothendieck topology $\mathbf{C}$ on a partially ordered set $(P, \leq)$; write $\mathscr{A} P)$ for the set of all sieves on $P$. A sieve $I$ will be said to cover an element $p \in P$ if $I$ includes a cover of $p$. Call $I$ C-closed if it contains every element of $P$ that it covers, i.e. if

$$
\exists S \in \mathbf{C}(p)(S \subseteq I) \rightarrow p \in I
$$

It is easy to see that the intersection of an arbitrary family of $\mathbf{C}$-closed sieves is a $\mathbf{C}$-closed sieve, so that for each sieve $I$ in $P$ there is a least $\mathbf{C}$-closed sieve $\tilde{I}$ containing $I$-the $\mathbf{C}$-closure of $I$. The operation of $\mathbf{C}$-closure satisfies two of the Kuratowski closure conditions above, namely $I \subseteq \tilde{I}$ and $\tilde{\tilde{I}}=I$, but instead of distributing over union it can be shown to distribute over intersection:

$$
\overline{I \cap J}=\tilde{I} \cap \widetilde{J} .
$$

It is worth noting that the characteristic properties of closure operations associated with the notion of covering were discovered almost exactly half a century after the isolation of the of the corresponding properties of closures derived from the notion of neighbourhood

I turn finally to the idea of a lattice of parts. Given a space or "whole" $S$, one expects the pertinent parts of $S$ to constitute a lattice $L$ under the primitive relation $\subseteq$ of inclusion. That is, for each pair $U, V$ of parts of $S$ we assume that there are parts $U \sqcup V, U \sqcap V$ 一the join and meet of $U, V$ 一which are the $\subseteq$ - least and $\subseteq$-largest parts, respectively, of $S$ containing, or, respectively, contained in, $U$ and $V$. We assume that $\varnothing$ and $S$ are the least and largest parts of $S$, 
respectively. We also suppose that for each part $U$ we are furnished with a part $U^{*}$ for which $U \sqcap U^{*}=\varnothing$ : $U^{*}$ is the designated part of $S$ "outside" $U$. We suppose that the operation * is inclusion inverting: $U \subseteq V \rightarrow V^{*} \subseteq U^{*}$. We shall call the structure $\mathbf{S}=(S, L)$ a mereotic space: $S$ is the whole, and the members of $L$ the parts, of $\mathbf{S}$.

Suppose now that we are given a number of primitive attributes $A, B$ ("red", "hard", etc.) that may be possessed by the parts of $\mathbf{S}$. Each part $U$ is assigned a collection $\mathbf{A}(U)$ of attributes possessed by it: we assume that the possession of primitive attributes is persistent in the sense that $U \subseteq V \rightarrow \mathbf{A}(V) \subseteq \mathbf{A}(U)$. The relation of possession is extended to compound attributes $\varphi$ in the following way, writing $U \Vdash \varphi$ for " $U$ possesses $\varphi$ ":

$$
\begin{aligned}
& U \Vdash A \text { iff } A \in \mathbf{A}(U) \text { for primitive } A ; \\
& U \Vdash \varphi \wedge \psi \text { iff } U \Vdash \varphi \text { and } U \Vdash \psi ; \\
& U \Vdash \varphi \vee \psi \text { iff } V \Vdash \varphi \text { and } W \Vdash \psi \text { for some parts } V, W \text { such that } U=V \sqcup W \text {; } \\
& U \Vdash \neg \varphi \text { iff } V \Vdash \varphi \rightarrow V \subseteq U^{*} \text { for all } V \text {. }
\end{aligned}
$$

Call an attribute $\varphi$ persistent in $\mathbf{S}$ if for all parts $U, V$,

$$
U \Vdash \varphi \text { and } V \subseteq U \rightarrow V \Vdash \varphi .
$$

Persistence of arbitrary attributes in $\mathbf{S}$ is ensured by the distributivity of $L$ : that is, the truth of the law

$$
x \wedge(y \vee z)=(x \wedge y) \vee(x \wedge z)
$$

The two conditions are equivalent provided that corresponding to each part $U$ there is an attribute $A_{U}$ such that $X \Vdash A_{U} \Leftrightarrow X \subseteq U$ for all parts $X$. If we think of the relation $\Vdash$ as a kind of covering relation between parts and 
attributes (so $U \Vdash \varphi$ is understood as asserting that $\varphi$ covers $U$, then persistence of arbitrary attributes in $\mathbf{S}$ may be seen as amounting to the satisfaction of the second postulate of a Grothendieck topology: the restriction of a cover is a cover.

There are natural mereotic spaces $\mathbf{S}$ arising in connection with proximity structures in which certain attributes $\varphi$ are not persistent: in fact, $\varphi$ may be possessed by the whole space but not by certain parts. In this case attributes of the whole are not reflected in all of its parts.

A proximity structure is a set $S$ equipped with a reflexive symmetric binary relation $\simeq$. For each $x \in S$ we define the blob at $x, B_{x}$, by

$$
B_{x}=\{y \in S: x \simeq y\} .
$$

Unions of blobs are called parts of $S$. We obtain a mereotic space $(S, \operatorname{Part}(S))$-called a proximity space-in which the join operation is settheoretic union, the meet of two parts of $S$ is the union of all blobs included in their intersection, and for $U \in \operatorname{Part}(S)$,

$$
U^{*}=\{y \in S: \forall x \in U . x \neq y\} .
$$

I conclude with a simple example of a nonpersistent proximity space. Take $S$ to be the unit circle and the blob at any point $x \in S$ to be the quadrant centred on the radius vector passing through $x$. Now assign the attributes red and black to the parts of $S$ by colouring the first and third quadrants red and the second and fourth black. Then $S$ possesses the attribute red $\vee$ black. On the other hand, if $U$ is (either) semicircle with diameter at 45 degrees to the $x$ axis, then clearly $U$ fails to possess red $\vee$ black, since it cannot be decomposed into two parts possessing the respective attributes red and black. A similar argument shows that $S$, but not $U$, possesses the attribute red $\vee \neg$ red. On the other hand, $U$ possesses the attribute $\neg \neg($ red $\vee$ black), since it can be shown that, for any part $U$ of a proximity space, and any attribute $\varphi$, 


$$
U \Vdash \neg \neg \varphi \leftrightarrow \exists V \supseteq U . V \Vdash \varphi .
$$

In a proximity space, in other words, a part $U$ possesses the double negation of an attribute exactly when $U$ is itself a part of a larger part possessing the attribute. Accordingly, while arbitrary attributes may not be persistent, double negated attributes always persist.

\section{References}

Bell, J. L. and Machover, M.: 1977. A Course in Mathematical Logic. NorthHolland, Amsterdam.

Bell, J. L. and Slomson, A. B. : 1969, Models and Ultraproducts: An Introduction. North-Holland, Amsterdam.

Bourbaki, N. : 1990, Elements of Mathematics: Algebra II. Springer-Verlag, New York.

Edwards, R. E.: 1965, Functional Analysis. Holt, Rinehart and Winston, New York.

Gillman, L. and Jerison, M. : 1960, Rings of Continuous Functions. Van Nostrand, New York.

Johnstone, P. T.: 1982, Stone Spaces. Cambridge University Press, Cambridge, U.K.

Lawvere, F. W. and Schanuel, S.: 1997, Conceptual Mathematics. Cambridge University Press, Cambridge, U.K.

Loomis, L. H.: 1953, Abstract Harmonic Analysis. Van Nostrand, New York.

Mac Lane, S. and Moerdijk, I.: 1992, Sheaves in Geometry and Logic. SpringerVerlag, New York.

Mulvey, C. J.: 1979, 'Representations of Rings and Modules', in M.P. Fouman, C.J. Mulvey and D.S. Scott, eds. , Applications of Sheaves, Lecture Notes in Mathematics 753, Springer-Verlag, New York, pp. 542-585.

Robinson, A.: 1996, Non-Standard Analysis. Princeton University Press, Princeton, N.J., U.S.A. 
Spivak, M.: 1979, Differential Geometry, Publish or Perish Press, Waltham, Mass., U.S.A. 